\title{
Celastrol inhibits the HIF-1 $\alpha$ pathway by inhibition of mTOR/p70S6K/eIF4E and ERK1/2 phosphorylation in human hepatoma cells
}

\author{
JUAN MA ${ }^{1,2 *}$, LI ZHUO HAN ${ }^{4 *}$, HE LIANG $^{1,2}$, CHUNLIU MI ${ }^{2,3}$, HUI SHI $^{2,3}$, \\ JUNG JOON LEE ${ }^{2}$ and XUEJUN JIN ${ }^{1,2}$
}

\begin{abstract}
${ }^{1}$ Key Laboratory of Natural Resources of Changbai Mountain and Functional Molecules, Ministry of Education, College of Pharmacy; ${ }^{2}$ Molecular Cancer Research Center; ${ }^{3}$ Key Laboratory of Natural Resources of Changbai Mountain and Functional Molecules, Ministry of Education, Department of Chemistry, Yanbian University, Yanji, Jilin 133002;

${ }^{4}$ Department of Pharmacy, Jilin Province People's Hospital, Changchun, Jilin 130021, P.R. China
\end{abstract}

Received March 11, 2014; Accepted May 6, 2014

DOI: $10.3892 / o r .2014 .3211$

\begin{abstract}
Hypoxia-inducible factor-1 (HIF-1) is the central mediator of cellular responses to low oxygen and vital to many aspects of cancer biology. In a search for HIF-1 inhibitors, we identified celastrol as an inhibitor of HIF-1 activation from Tripterygium wilfordii. In the present study, we demonstrated the effect of celastrol on HIF-1 activation. Celastrol showed a potent inhibitory activity against HIF-1 activation induced by hypoxia in various human cancer cell lines. This compound markedly decreased the hypoxia-induced accumulation of HIF-1 $\alpha$ protein dose-dependently, whereas it did not affect the expressions of HIF-1 $\beta$ and topoisomerase-I (topo-I). Furthermore, celastrol prevented hypoxia-induced expression of HIF-1 target genes for vascular endothelial growth factor (VEGF) and erythropoietin (EPO). Further analysis revealed that celastrol inhibited HIF-1 $\alpha$ protein synthesis, without affecting the expression level of HIF-1 $\alpha$ mRNA or degrada-
\end{abstract}

Correspondence to: Professor Jung Joon Lee, Molecular Cancer Research Center, Yanbian University, Yanji, Jilin 133002, P.R. China E-mail: jjlee0908@gmail.com

Professor Xuejun Jin, Key Laboratory of Natural Resources of Changbai Mountain and Functional Molecules, Ministry of Education, College of Pharmacy, Yanbian University, Yanji, Jilin 133002, P.R. China

E-mail: xjjin@ybu.edu.cn

${ }^{*}$ Contributed equally

Abbreviations: HIF-1, hypoxia-inducible factor-1; VEGF, vascular endothelial growth factor; topo-I, topoisomerase-I; EPO, erythropoietin; ERK1/2, extracellular signal-regulated kinase-1/2; mTOR, mammalian target of rapamycin; eIF4E, eukaryotic initiation factor 4E; p70S6K, ribosomal protein S6 kinase; HRE, hypoxia response element

Key words: celastrol, HIF-1 $\alpha$, translation, antitumor tion of HIF-1 $\alpha$ protein. Markedly, we found that suppression of HIF-1 $\alpha$ accumulation by celastrol correlated with strong dephosphorylation of mammalian target of rapamycin (mTOR) and its effectors, ribosomal protein S6 kinase (p70S6K) and eukaryotic initiation factor 4E (eIF4E) and extracellular signalregulated kinase (ERK), pathways known to regulate HIF-1 $\alpha$ expression at the translational level. In vivo studies further confirmed the inhibitory effect of celastrol on the expression of HIF-1 $\alpha$ proteins, leading to a decreased growth of Hep3B cells in a xenograft tumor model. Our data suggested that celastrol is an effective inhibitor of HIF-1 and provide new perspectives into the mechanism of its anticancer activity.

\section{Introduction}

Hypoxia is an important micro-environmental factor in promoting tumor progression. In hypoxia, several transcription factors are induced to respond to the decreased oxygen level. Among these transcription factors, hypoxia-inducible factor-1 (HIF-1) is one of the most important factors that play a critical role in controlling oxygen delivery and metabolic adaptation to hypoxic conditions. HIF-1 is a heterodimeric protein consisting of a constitutively expressed HIF- $1 \beta$ subunit, known as the aryl hydrocarbon receptor nuclear translocator and HIF-1 $\alpha$ subunit, which are basic helixloop-helix-PAS domain proteins; only HIF-1 $\alpha$ is regulated by the oxygen tension $(1,2)$. The regulation of HIF-1 $\alpha$ occurs at the posttranslational level involving modifications of hydroxylation, acetylation and phosphorylation (3-7). Under normoxic conditions, prolyl hydroxylases (PHDs) hydroxylate the cite-specific proline residues of HIF- $1 \alpha$ in a reaction that uses $\mathrm{O}_{2}$ as a substrate. The modified HIF-1 $\alpha$ interacts with Von Hippel-Lindau (VHL), which is part of the E3 ubiquitin ligase complex targeting HIF-1 $\alpha$ for $26 \mathrm{~S}$ proteasomal degradation. Under hypoxia, HIF-1 $\alpha$ is stabilized due to the lack of $\mathrm{O}_{2}$ and dimerizes with HIF-1 $\beta$ interacting with the co-activator $\mathrm{CBP} / \mathrm{p} 300$ to bind to the hypoxia response element (HRE; 5'-GACGTG-3') on the promoter region in various target genes $(1,2)$. 
HIF-1 plays a central role in tumor progression and angiogenesis in vivo. For instance, it can be activated by oncogenic mutations of PTEN, VHL, the RAS/mitogen-activated protein kinase (MAPK) pathway and the phosphorylation of phosphatidylinositol 3-kinase (PI3K)/Akt/mammalian target of rapamycin (mTOR) pathway. Furthermore, HIF-1 $\alpha$ is also stabilized by reactive oxygen species (ROS), which block PHD activities (8). To date, HIF-1 is known to transcriptionally upregulate $>100$ genes $(9,10)$. Exposure to a variety of growth factors has also been shown to increase HIF-1 activity in normoxic and hypoxic conditions. HIF-1 $\alpha$ overexpression is associated with increased mortality in patients with various tumors; this association is primarily based on the HIF-1-mediated regulation of genes that play pivotal roles in the central features of cancer pathogenesis such as angiogenesis, invasion, metastasis and anti-apoptosis. All of these activities make the HIF-1 transcription factor an attractive target for the development of new anticancer therapeutics (11-13).

As part of our continuing search for HIF-1 $\alpha$ inhibitors from natural products, we identified celastrol, a quinone methide triterpene, as a pharmacologically active compound in Tripterygium wilfordii Hook F root extracts. Celastrol has been widely used to treat autoimmune diseases, chronic inflammation, neurodegenerative diseases as well as several types of cancer (14). However, the mechanism by which celastrol inhibits HIF-1-mediated tumor growth is not fully understood. In present study, we found that celastrol inhibited HIF-1 activation. This compound rapidly downregulates not only HIF-1 $\alpha$, by decreasing its protein synthesis without affecting mRNA levels or protein degradation, but also the expression of HIF target genes such as vascular endothelial growth factor (VEGF) and erythropoietin (EPO), which are essential for tumor growth. The HIF-1 activation-inhibitory effects of this compound were associated with the suppression of mTOR/ribosomal protein S6 kinase (p70S6K)/eukaryotic initiation factor 4E (eIF4E) and extracellular signal-regulated kinase (ERK) signaling pathways. We further confirmed our in vitro observations by showing profound antitumor activity of celastrol in a murine xenograft model with no apparent toxicity to the animals.

\section{Materials and methods}

Cell culture and reagents. Hep3B, SK-Hep1, and HeLa cells were grown in DMEM with penicillin (100 U/ml)-streptomycin (100 U/ml) (Invitrogen, Carlsbad, CA, USA) and 10\% heat-inactivated fetal bovine serum (Hyclone, Logan, UT, USA). All cells were purchased from American Type Culture Collection (ATCC, Manassas, VA, USA). MG-132 and cycloheximide (CHX) were from Sigma (St. Louis, MO, USA). The hypoxic culture was kept in a gas-controlled chamber (Thermo Electron Corp., Marietta, $\mathrm{OH}$, USA) maintained at $1 \% \mathrm{O}_{2}, 94 \%$ $\mathrm{N}_{2}$, and $5 \% \mathrm{CO}_{2}$ at $37^{\circ} \mathrm{C}$. In some experiments, cobalt chloride was used to induce hypoxia mimicking conditions. Cobalt chloride was reported as a widely used mimetic of hypoxia in a large range of cells; the molecule is known to inhibit prolyl hydroxylases leading to HIF-1 $\alpha$ stabilization (15). Celastrol was isolated from $T$. kirilowii and its structure is shown in Fig. 1A. The purity of celastrol was $>98 \%$ in the HPLC analysis.
Plasmids, transfections and luciferase reporter assay. The ability of the compound to inhibit hypoxia inducible factor was determined by HRE-dependent reporter assay as previously described (16). In brief, at 50-80\% confluence, Hep3B cells, which were co-transfected with the vectors for pGL3-HRE-Luciferase plasmid containing six copies of HREs derived from the human VEGF gene and with pRL-CMV (Promega, Madison, WI, USA) using Lipofectamine plus reagent (Invitrogen, Carlsbad, CA, USA). Following $24 \mathrm{~h}$ incubation, the cells were treated with various concentrations of celastrol and incubated for $16 \mathrm{~h}$ in hypoxia. Luciferase assay was performed using dual-luciferase reporter assay system according to the instructions of the manufacturer (Promega). Luciferase activity was determined in Microlumat plus luminometer (EG\&G Berthold, Bad Wildbad, Germany) by injecting $100 \mu \mathrm{l}$ of assay buffer containing luciferin and measuring light emission for $10 \mathrm{sec}$. The results were normalized to the activity of renilla expressed by cotransfected Rluc gene under the control of a constitutive promoter.

Measurement of in vitro invasion and cell viability. The ability of cells to invade through Matrigel-coated filters (invasion) was determined using a modified 24-well Boyden chamber (Corning Costar, Cambridge, MA, USA; $8 \mu \mathrm{m}$ pore size) as previously described (17). Hep3B cells were seeded at a density of $5 \times 10^{4}$ cells in $100 \mu \mathrm{l}$ DMEM containing $10 \%$ FBS in the upper compartment of transwell. To determine the effect of celastrol, various concentrations of celastrol were added to the lower or upper compartment of transwell. After incubation for $24 \mathrm{~h}$ at $37^{\circ} \mathrm{C}$ in $5 \% \mathrm{CO}_{2}$, the cells that did not penetrate the filter were completely wiped out with a cotton swab, and the cells that had migrated to the lower surface of the filter were fixed, stained and counted in 5 randomly selected microscopic fields (x100) per filter. Cell viability was measured by an MTT [3-(4,5-dimethylthiazol-2-yl)-2,5-diphenyltetrazolium bromide] assay (Sigma). Briefly, untreated cells or treated cells with celastrol in a 96-well plate were incubated for $24 \mathrm{~h}$ followed by the addition of MTT to the cells. Optical densities were determined on a microplate reader (Molecular Devices, Sunnyvale, CA, USA).

Western blot analysis. Whole-cell extracts were obtained by lysing cells in ice-cold lysis buffer (50 mM Tris- $\mathrm{HCl}, \mathrm{pH} 7.5$, $1 \%$ Nonidet P-40, $1 \mathrm{mM}$ sulfonyl fluoride) supplemented with the protease inhibitor cocktail (BD Biosciences, San Diego, CA, USA). HIF-1 $\alpha$ protein was analyzed in nuclear extracts prepared from cells using NE-PER reagent (Pierce, Rockford, IL, USA), according to the instructions of the manufacturer. An aliquot of protein extracts was used to determine protein concentration by the Bradford method. Fifty micrograms of whole-cell extracts or $30 \mu \mathrm{g}$ of nuclear extract protein per lane was separated by SDS-polyacrylamide gels and followed by transferring to a polyvinylidene difluoride membrane (Millipore, Bedford, MA, USA). The membrane was blocked with $5 \%$ skim milk, and then incubated with the corresponding antibody. Antibody for HIF-1 $\alpha$ was obtained from BD Biosciences (1:250). Antibodies for mTOR, phospho (Ser2448)-specific mTOR, p70S6K, phospho (Thr389)specific p70S6K, ERK1/2, phospho (Thr202/Tyr204)-specific ERK1/2, eIF4E, phospho (Ser209)-specific eIF4E, p38, 
A

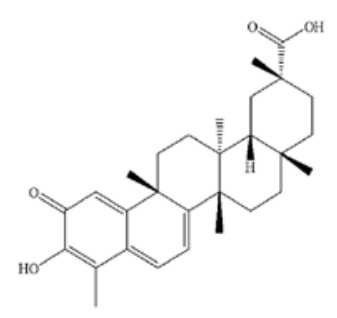

B

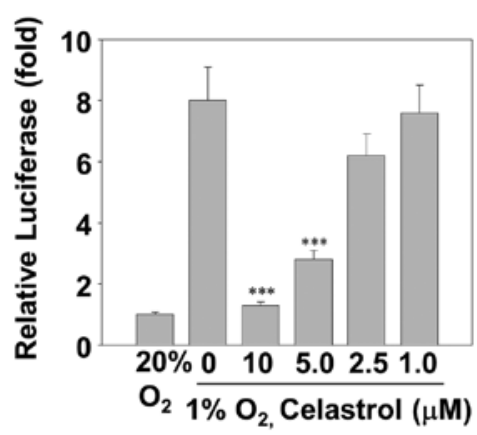

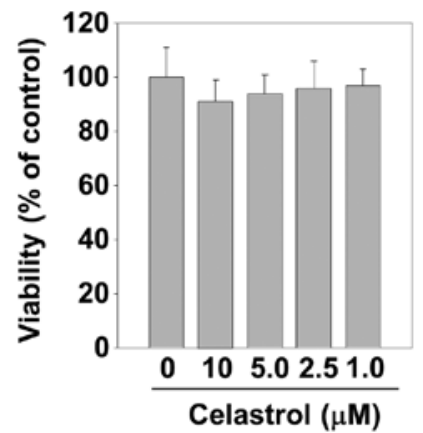

Celastrol $(\mu \mathrm{M})$
C
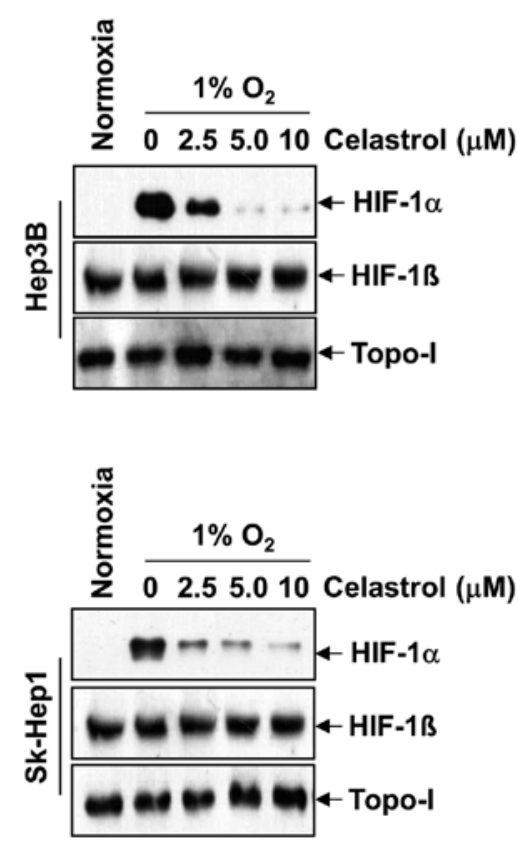
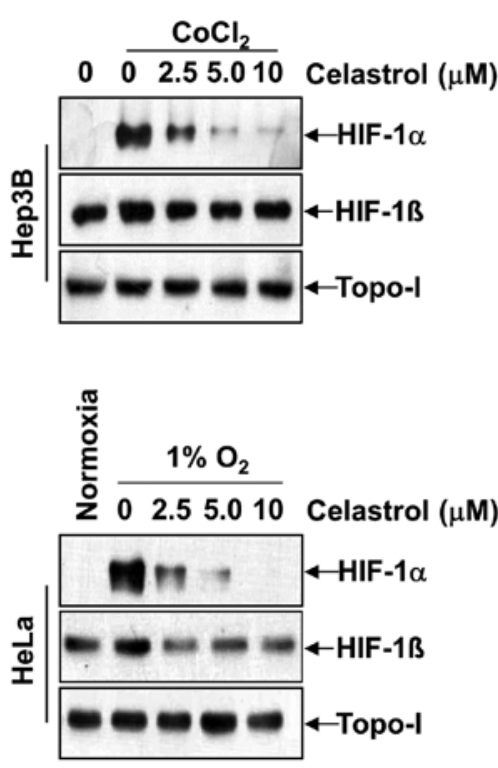

Figure 1. Effect of celastrol on the expression of HIF-1 $\alpha$ protein. (A) Chemical structure of celastrol. (B) Effect of celastrol on HRE-mediated reporter gene expression under hypoxia. Hep3B cells were transiently co-transfected with a pGL3-HRE-Luciferase and pRL-CMV vectors. Following 24 h incubation, the cells were incubated under hypoxia in the absence or presence of the indicated concentrations of celastrol. Luciferase activities were determined as described in Materials and methods. Data are presented as mean \pm standard deviation of three independent experiments. ${ }^{* * *} \mathrm{P}<0.001$ significant with respect to control. Hep3B cells were treated with the indicated concentrations of celastrol. After $24 \mathrm{~h}$ incubation, cell viability was determined by MTT assays. (C) Cancer cells, Hep3B, SK-Hep1 and HeLa, were incubated under normoxia or hypoxia for $12 \mathrm{~h}$, in the absence or presence of the indicated concentrations of celastrol. Whole-cell lysates for HIF-1 $\beta$ and nuclear extract for HIF-1 $\alpha$ were analyzed by western blotting. The same blot was reprobed with an anti-topo-I antibody as a loading control. Hep3B cells were pretreated with the indicated concentrations of celastrol for $30 \mathrm{~min}$ and treated with $\mathrm{CoCl}_{2}(200 \mu \mathrm{M})$. After $12 \mathrm{~h}$ incubation, the whole-cell lysates for HIF-1 $\beta$ and nuclear extract for HIF-1 $\alpha$ were analyzed by western blotting. The same blot was reprobed with an anti-topo-I antibody as a loading control.

phospho-specific p38, SAPK/JNK and phospho-specific SAPK/JNK were purchased from Cell Signaling Technology (Beverly, MA, USA). Antibodies for HIF-1 $\beta$ and topo-I were obtained from Santa Cruz Biotechnology (Santa Cruz, CA, USA). Antibody for $\alpha$-tubulin was from Sigma. After binding of an appropriate secondary antibody coupled to horseradish peroxidase, proteins were visualized by enhanced chemiluminescence according to the instructions of the manufacturer (Amersham Pharmacia Biotech, Buckinghamshire, UK).

VEGF ELISA. Hep3B cells were plated in 96-well plates at a density of $1 \times 10^{5}$ cells/well and treated with various concentrations of celastrol for $16 \mathrm{~h}$ under normoxic or hypoxic conditions. The VEGF levels in the culture supernatant were determined by ELISA using the Duo-Set ELISA development kit (R\&D Systems, Inc., Minneapolis, MN, USA), according to the manufacturer's instructions.

RT-PCR analysis. Total RNA from Hep3B cells was obtained using RNA Mini kit (Qiagen, Valencia, CA, USA). Total RNA (2 $\mu \mathrm{g}$ ) was used to perform reverse transcription-PCR (RT-PCR) using RT-PCR kit (Invitrogen, Carlsbad, CA, USA) according to the manufacturer's protocol. The PCR primers were: VEGF, 5'-GCTCTACCTCCACCATGCCAA-3' (sense) and 5'-TGGAAGATGTCCACCAGGGTC-3' (antisense); EPO, 5'-CACTTTCCGCAAACTCTTCCG-3' (sense) and 5'-GTCA CAGCTTGCCACCTAAG-3' (antisense); HIF-1 $\alpha$, 5'-CTCA AAGTCCGACAGCCTCA-3' (sense) and 5'-CCCTGCAGT AGGTTTCTGCT-3' (antisense); GAPDH, 5'-ACCACAGTC CATGCCATCAC-3' (sense) and 5'-TCCACCACCCTGTT 
GCTGTA-3' (antisense). The oligonucleotide sequences of the reaction products were confirmed by sequencing.

In vivo xenograft assay. All surgical procedures and careful handling of the animals were in accordance with IACUC guidelines. Six-week-old specific pathogen-free Crj:BALB/c nu/nu female athymic nude mice (Vital River, China) were randomly assigned to three groups, each of which consisted of five mice ( $\mathrm{n}=5$ per group), and were then subcutaneously inoculated with $0.2 \mathrm{ml}$ of Hep3B cells $\left(5 \times 10^{7}\right.$ cells $\left./ \mathrm{ml}\right)$ in the right flank region. Celastrol, dissolved in DMSO, was administered orally three times a week for 35 days at a dose of 3 and $10 \mathrm{mg} / \mathrm{kg}$ body weight starting from the ten days post cell implantation with mice. Tumor volume was calculated every five days using the equation: (Length $\mathrm{x}$ (width)2)/2. Tumors were harvested $4 \mathrm{~h}$ after the last treatment, followed by homogenizing in RIPA for western blotting analysis.

Statistical analysis. All values are expressed as mean \pm SD. A comparison of the results was performed with one-way ANOVA and Tukey's multiple comparison tests (Graphpad Software, Inc., San Diego, CA, USA). Statistically significant differences between groups were defined as P-values $<0.01$.

\section{Results}

Celastrol inhibits HIF-1 $\alpha$ expression in tumor-derived cells. To investigate whether celastrol inhibited HIF-1 $\alpha$ transcriptional activation, we transfected Hep3B cells with a luciferase reporter gene driven by six specific HREs. A substantial increase of luciferase activity was observed in cells cultured in hypoxic conditions, whereas celastrol dose-dependently inhibited hypoxia-induced luciferase activity (Fig. 1B, left panel). Given that the inhibition of HIF-1 $\alpha$ transcriptional activation might be correlated with celastrol-induced cytotoxicity, parallel studies of cell viability were performed (Fig. 1B, right panel). After the Hep3B cells were treated with celastrol (up to $10 \mu \mathrm{M}$ ) for $24 \mathrm{~h}$, no significant alteration of cell viability was observed relative to the untreated control group.

To explore the mechanism underlying celastrol activity, we investigated its effect on HIF-1 $\alpha$ protein levels. In Hep3B cells, HIF-1 $\alpha$ protein is undetectable under normoxia, whereas it is stabilized under hypoxia or in the presence of $\mathrm{CoCl}_{2}$ and becomes readily detectable by western blotting. Following 12-h treatment, celastrol exerted dose-dependent inhibition of HIF-1 $\alpha$ protein levels induced by hypoxia or $\mathrm{CoCl}_{2}$ in $\mathrm{Hep} 3 \mathrm{~B}$ cells. (Fig. 1C, top two panels). In contrast to the decrease of HIF-1 $\alpha$ levels, celastrol had almost no effect on the levels of HIF-1 $\beta$ and topo-I proteins. Next, in order to address whether the inhibition of HIF-1 $\alpha$ by celastrol was cell line-specific, we extended these studies to a diverse set of tumor cell lines with tissues of various origins, including the hepatic cancer cell lines SK-Hep1 and epithelial cervical cancer cell lines HeLa cells. Fig. 1C shows that, under hypoxic conditions, HIF-1 $\alpha$ accumulation was strongly suppressed by celastrol in all cell lines.

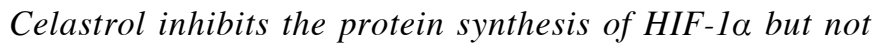
its degradation. Generally, the accumulation of HIF-1 $\alpha$ is dependent on the balance between its protein synthesis and
A

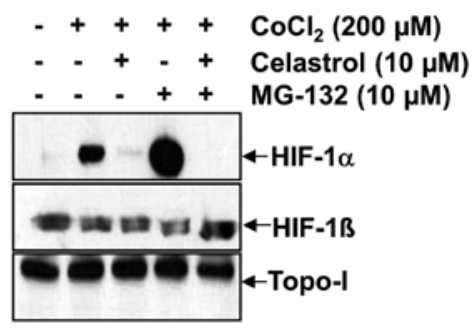

B

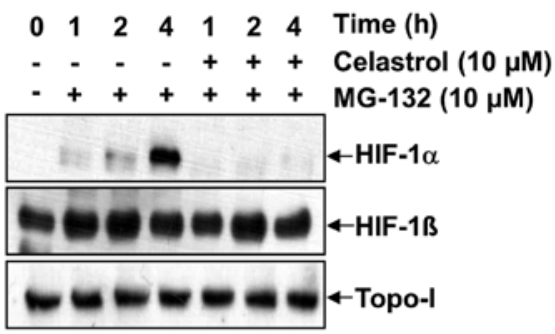

C

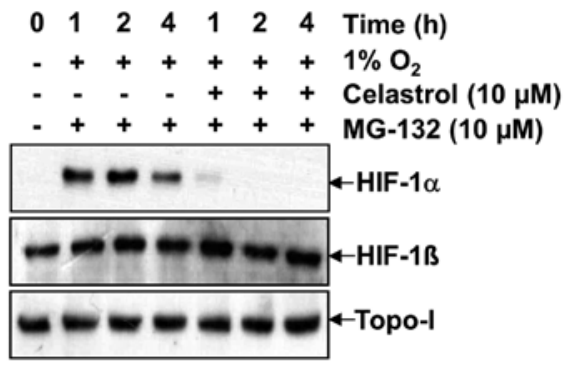

Figure 2. Effect of celastrol on HIF-1 $\alpha$ protein accumulation induced by proteasome inhibition. (A) Proteasome inhibitor MG-132 $(10 \mu \mathrm{M})$ was added to Hep3B cells for 30 min prior to the treatment of celastrol $(10 \mu \mathrm{M})$ and then the cells were incubated in the presence of $\mathrm{CoCl}_{2}(200 \mu \mathrm{M})$ for $12 \mathrm{~h}$ (B) Hep3B cells were treated with MG-132 $(10 \mu \mathrm{M})$ for 1,2 , and $4 \mathrm{~h}$ in the presence or absence of celastrol $(10 \mu \mathrm{M})$. (C) Proteasome inhibitor MG-132 $(10 \mu \mathrm{M})$ was added to Hep3B cells for 30 min prior to the treatment of celastrol $(10 \mu \mathrm{M})$ and then the cells were incubated under hypoxia for $12 \mathrm{~h}$. Cells were harvested, and whole-cell lysates for HIF-1 $\beta$ and nuclear extract for HIF-1 $\alpha$ were analyzed by western blotting. The same blot was reprobed with an anti-topo-I antibody as a loading control.

degradation. To specifically investigate whether celastrol modulates HIF- $1 \alpha$ protein synthesis, we used the proteasome inhibitor MG-132 to prevent HIF-1 $\alpha$ degradation. This allowed us to observe HIF-1 $\alpha$ stabilization and accumulation following de novo protein synthesis. Due to rapid proteosomal destruction and undetectable HIF-1 $\alpha$ levels in normoxic cells, the accumulation rate of HIF-1 $\alpha$ via proteosomal inhibition reflects the synthesis rate of the protein (18). As shown in Fig. 2A, B and C, HIF-1 $\alpha$ rapidly accumulated over a period of $4 \mathrm{~h}$ in the presence of MG-132 under both normoxia and hypoxia, as well as in the presence of $\mathrm{CoCl}_{2}$. In contrast, co-treatment with celastrol and MG-132 resulted in a much slower rate of HIF-1 $\alpha$ accumulation (compare lane 4 with lane 5 for $\mathrm{CoCl}_{2}$ in Fig. 2A, lanes 2-4 with lanes 5-7 under normoxia in Fig. 2B, and lanes 2-4 with lanes 5-7 under hypoxia in Fig. 2C). No effects were observed on HIF-1 $\beta$ and topo-I. These results indicate that HIF-1 $\alpha$ protein synthesis in Hep3B cells is markedly impaired in the presence of celastrol. To address the effect of celastrol on HIF-1 $\alpha$ protein stability, 
A

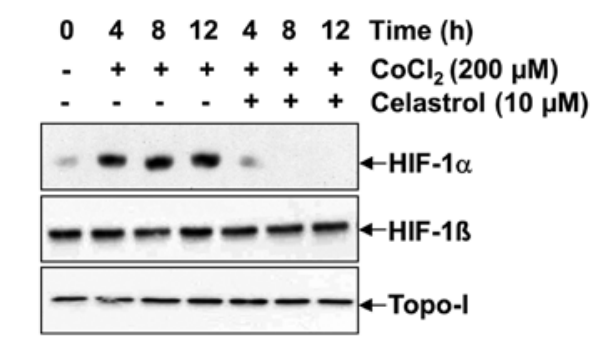

B

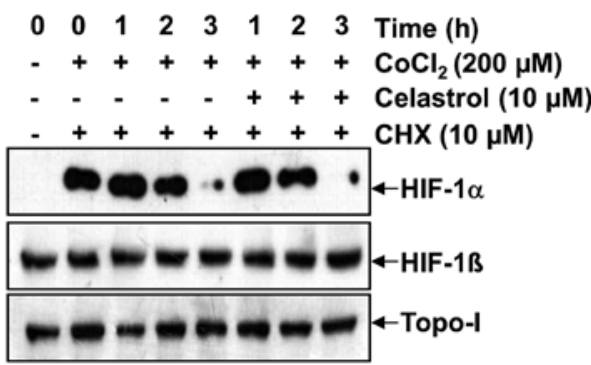

Figure 3. Effect of celastrol on proteasomal degradation of HIF-1 $\alpha$. (A) Hep3B cells were pretreated for 30 min with celastrol $(10 \mu \mathrm{M})$, and then stimulated with $\mathrm{CoCl}_{2}(200 \mu \mathrm{M})$ for the indicated times. Subsequently, whole-cell lysates for HIF-1 $\beta$ and nuclear extract for HIF-1 $\alpha$ were analyzed by western blotting. The same blot was reprobed with an anti-topo-I antibody as a loading control. (B) Hep3B cells were incubated in the presence of $\mathrm{CoCl}_{2}$ $(200 \mu \mathrm{M})$ for $4 \mathrm{~h}$. CHX $(10 \mu \mathrm{M})$ and celastrol $(10 \mu \mathrm{M})$ were then mixed with culture media. After 1,2, or $3 \mathrm{~h}$ following the addition of CHX, cells were harvested and whole-cell lysates for HIF-1 $\beta$ and nuclear extract for HIF-1 $\alpha$ were analyzed by western blotting. The same blot was reprobed with an anti-topo-I antibody as a loading control.

the protein translation inhibitor $\mathrm{CHX}$ was used to prevent de novo HIF-1 $\alpha$ protein synthesis. Accordingly, we determined whether celastrol affects HIF- $1 \alpha$ protein stability by the addition of $200 \mu \mathrm{M} \mathrm{CoCl}_{2}$ (Fig. 3). HIF-1 $\alpha$ accumulation increased in a time-dependent manner, but the addition of celastrol resulted in the significant abrogation of HIF-1 $\alpha$ accumulation at every time point (Fig. 3A). To determine whether HIF-1 $\alpha$ protein half-life was affected by celastrol, we firstly induced HIF-1 $\alpha$ accumulation in the presence of $200 \mu \mathrm{M} \mathrm{CoCl}_{2}$ for $4 \mathrm{~h}$, and then added $\mathrm{CHX}$ alone or in combination with celastrol. In the presence of $\mathrm{CHX}$, HIF-1 $\alpha$ levels rapidly declined in both the celastrol-untreated and celastrol-treated cells, showing a half-life was $\sim 1.5 \mathrm{~h}$ in Hep3B cells. These results indicated that celastrol did not modify the degradation rate of HIF-1 $\alpha$ (Fig. 3B, lanes 3-5 with lanes 6-8).

To determine whether HIF-1 $\alpha$ synthesis inhibition by celastrol was a downstream effect from decreased HIF-1 $\alpha$ gene transcription or HIF-1 $\alpha$ mRNA stability, we analyzed HIF-1 $\alpha$ mRNA levels by RT-PCR. Celastrol did not change HIF-1 $\alpha$ mRNA levels under either normoxia or hypoxia during $16 \mathrm{~h}$ of treatment (Fig. 5A). This suggests that celastrol-mediated decrease of HIF-1 $\alpha$ synthesis is due to downregulation of HIF-1 $\alpha$ mRNA translation.

Downregulation of $m T O R / p 70 S 6 K / e I F 4 E$ and ERK1/2 phosphorylation by celastrol correlates with inhibition of HIF-1a synthesis. To reveal the underlying mechanism by which
A

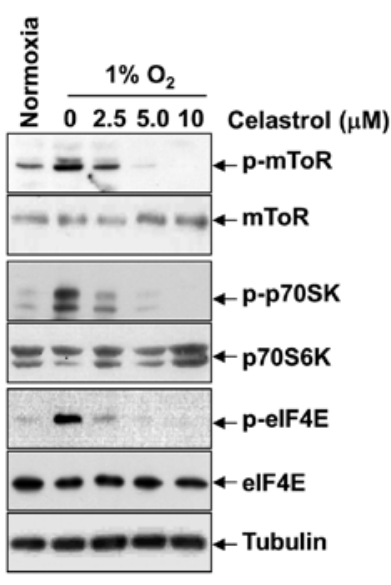

B

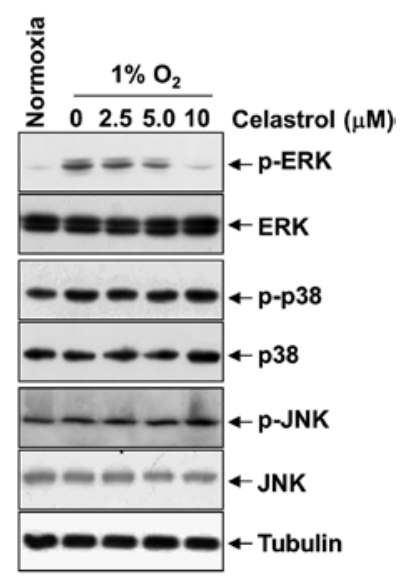

Figure 4. Effect of celastrol on mTOR/p70S6K/eIF4E and ERK/p38/JNK signaling. (A) Hep3B cells were incubated under normoxia or hypoxia for $12 \mathrm{~h}$, in the absence or presence of the indicated concentrations of celastrol. Phospho-mTOR, phospho-p70S6K, and phospho-eIF4E were detected by western blot analysis. The bottom represents the corresponding total protein to show the equal loading of cell lysates. Tubulin level was used as a loading control. (B) Hep3B cells were incubated under normoxia or hypoxia for $12 \mathrm{~h}$, in the absence or presence of the indicated concentrations of celastrol. Phospho-ERK1/2, phospho-p38, and phospho-JNK were detected by western blot analysis. The bottom represents the corresponding total protein to show the equal loading of cell lysates. Tubulin level was used as a loading control.

celastrol inhibits hypoxia-induced activation and translation of HIF-1 $\alpha$, we first examined the phosphorylation status of translation initiation factors such as mTOR, p70S6K, eIF4E, and ERK1/2 under hypoxia. As shown in Fig. 4A and B, in parallel with the alteration of HIF-1 $\alpha$ protein, celastrol dosedependently inhibited the expression of phospho-mTOR, phospho-p70S6K, phospho-eIF4E, and phospho-ERK1/2, induced by hypoxia; however, it had no inhibitory effect on phospho-p38 and phospho-JNK, and total protein levels for mTOR, p70S6K, eIF4E, ERK1/2, p38, and JNK. These findings were partially in line with a report showing that mTOR/p70S6K and ERK pathways are involved in HIF-1 $\alpha$ protein synthesis via functional activation of the translational regulatory protein eIF4E in various cells (19). All of the results consistently support the idea that celastrol inhibits hypoxia-induced activation of HIF-1 $\alpha$ through inhibition of mTOR/p70S6K/eIF4E and ERK signaling.

Celastrol decreases expression of HIF-1 $\alpha$ target genes and suppresses the invasiveness of tumor cells. The expression of VEGF and EPO, which are involved in tumor cell proliferation, angiogenesis, invasion and metastasis, is known to be regulated by HIF-1 $\alpha$ (11). We therefore examined whether celastrol can suppress the expression of these genes. VEGF and EPO mRNA levels were measured by RT-PCR analysis in Hep3B cells. Treatment of the cells with celastrol resulted in a dose-dependent inhibition of VEGF and EPO mRNA expression (Fig. 5A). The concentrations to inhibit the expression of HIF-1 $\alpha$ target genes were comparable with those of HIF-1 $\alpha$ protein accumulation. This result led us to measure the VEGF protein concentration in the culture supernatant by ELISA. Consistently, the hypoxic induction of secreted VEGF 
A

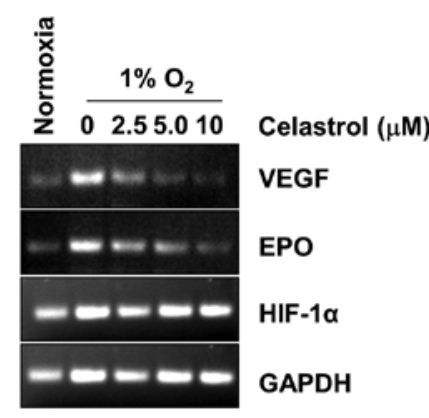

C

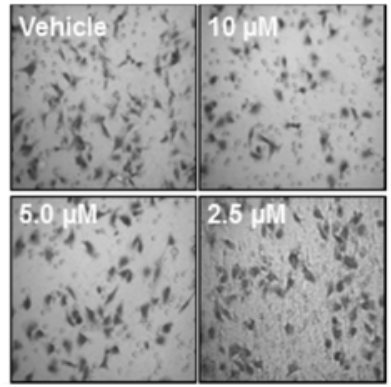

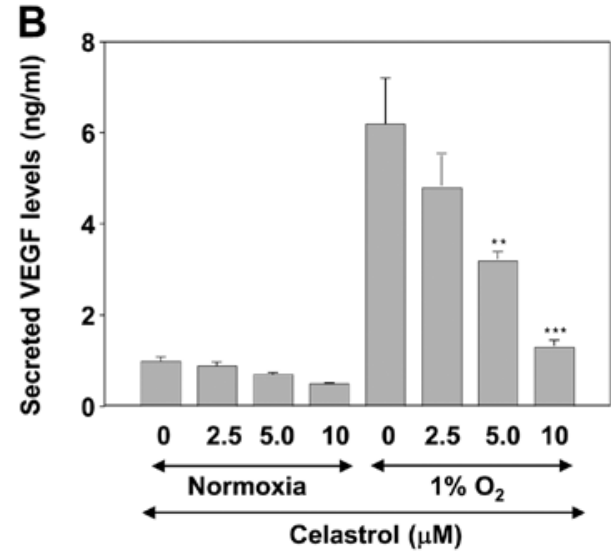

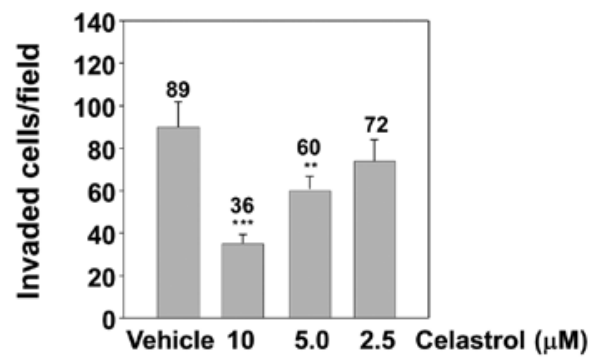

Figure 5. Effect of celastrol on the expression of HIF-1 $\alpha$ target genes and invasion in Hep3B cells. (A) Hep3B cells were incubated under normoxia or hypoxia for $16 \mathrm{~h}$ in the absence or presence of the indicated concentrations of celastrol. Total RNA was analyzed by RT-PCR as described in Materials and methods. (B) VEGF protein expression was evaluated by ELISA in culture supernatant of Hep3B cells after exposure to normoxia or hypoxia for $16 \mathrm{~h}$ in the presence or absence of the indicated concentrations of celastrol. Data are presented as mean \pm standard deviation of three independent experiments. ${ }^{* *} \mathrm{P}<0.01,{ }^{, * * *} \mathrm{P}<0.001$, significant with respect to control. (C) Cells that invaded through the pores in the Matrigel-coated filters were fixed, stained and counted in five random fields visualized by microscopy $(\mathrm{x} 100)$. Data are presented as mean \pm standard deviation of three independent experiments. ${ }^{* *} \mathrm{P}<0.01,{ }^{* * * *} \mathrm{P}<0.001$, significant with respect to control.
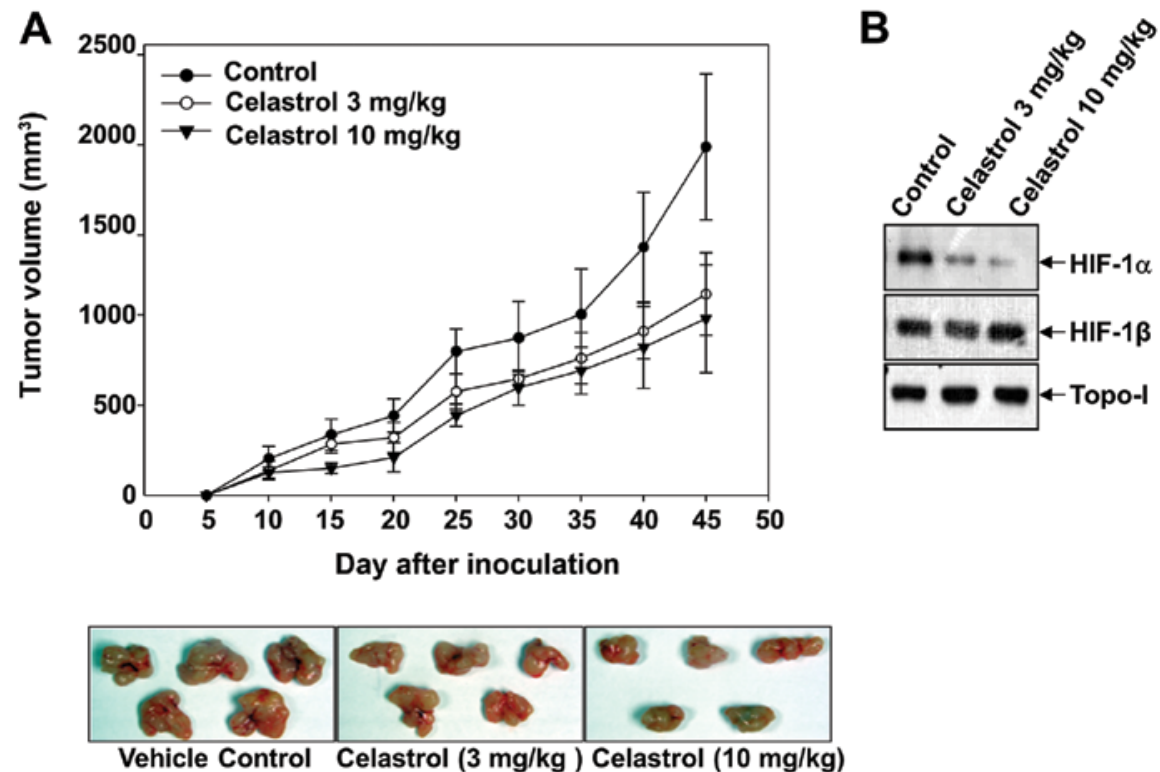

Figure 6. Effect of celastrol on Hep3B human xenograft model. (A) Hep3B cells were implanted s.c. in the left flanks of nude mice, which were administered p.o. three times a week with vehicle $(\mathrm{n}=5)$ or celastrol ( 3 or $10 \mathrm{mg} / \mathrm{kg}, \mathrm{n}=5)$ starting from day 10 . Tumor volume was calculated every five days using the equation: (Length $\mathrm{x}$ (width)2)/2. (B) Celastrol inhibited the expression of HIF-1 $\alpha$ in Hep3B xenograft. Hep3B xenograft tumor tissues were homogenized and the expression of HIF-1 $\alpha$ was detected by western blotting. Topo-I was used as a loading control.

protein was dose-dependently inhibited by celastrol (Fig. 5B). Reduced expression of VEGF and EPO might be responsible for diminished invasion of tumor cells in celastrol treatment.
Therefore, whether celastrol modulates invasion activity was examined in vitro with a Matrigel invasion assay. Hep3B cells were seeded in the top chamber of a Matrigel invasion chamber 
and were incubated with various concentrations of celastrol for $16 \mathrm{~h}$. The result showed that celastrol significantly decreased invasiveness compared to the vehicle control, accounting for the anti-invasive activity of celastrol (Fig. 5C).

Celastrol inhibits growth of Hep3B cells in a xenograft tumor model. Since celastrol suppresses both the expression of HIF-1 $\alpha$ target genes and the invasiveness of tumor cells, we next determined whether these results could be translated into an in vivo xenograft model. Hep3B cells were subcutaneously implanted in athymic nude mice, and the experimental mice were treated with celastrol ( 3 and $10 \mathrm{mg} / \mathrm{kg}$ ) 3 days a week until the end of the study. As expected, the administration of celastrol $(10 \mathrm{mg} / \mathrm{kg})$ significantly inhibited Hep3B tumor growth up to $52.9 \%$, compared to that of the vehicle-treated control group (Fig. 6A). Due to the key roles of HIF-1 $\alpha$ in tumor angiogenesis, we studied its expression in the tumors by western blotting. Consistent with the finding in cultured cells, celastrol significantly decreased the protein levels of HIF-1 $\alpha$ in the tumors, whereas no differences were observed in topo-I (Fig. 6B).

\section{Discussion}

Celastrol, a pharmacologically active compound in Tripterygium wilfordii Hook $\mathrm{F}$, has been used traditionally for the treatment of arthritis and other diseases (14). It has been reported that celastrol exerts potent anti-inflammatory activities in various experimental models and strong cytotoxicity against various cancer cell-lines; celastrol is also known to inhibit NF- $\kappa \mathrm{B}$ activation by directly targeting cysteine 179 in the IKK and this feature was supposed to contribute to its anti-inflammatory and antitumor activities through mechanisms associated with the inhibition of NF- $\kappa$ B (20). Previous studies have also shown that celastrol has antitumor and antiangiogenic effects in several tumor cells, and the underlying mechanisms were shown to be associated with their inhibitory effect on the proteasome, the AKT/mTOR/p70S6K pathway, and VEGF production and its receptor activity (21-23). These reports suggest that celastrol might be associated with the regulation of HIF-1. In the present study, we identified celastrol as a potent inhibitor of HIF-1 $\alpha$ activation and investigated how this compound suppressed HIF-1 $\alpha$ activation. The expression of HIF- $1 \alpha$ is tightly regulated through both protein degradation and protein synthesis. Our results showed that celastrol strongly inhibited HIF-1 $\alpha$ protein synthesis without affecting the expression level of HIF- $1 \alpha$ mRNA or degradation of HIF- $1 \alpha$ protein, indicating it acted as an inhibitor of HIF-1 $\alpha$ mRNA translation.

HIF-1 $\alpha$ protein translation has emerged as an important regulatory mechanism of HIF- $1 \alpha$-inhibitory compounds. In the present study, we found that treatment of Hep3B cells with celastrol suppressed phosphorylation of mTOR, phosphorylation of p70S6K, phosphorylation of eIF4E and phosphorylation of ERK1/2. Earlier studies have shown that HIF-1 $\alpha$ protein translation is mediated partly by regulation of free eIF4E levels through Akt/mTOR and ERK pathways (24-26). In the mTOR pathway, the mammalian target of rapamycin (mTOR), a $289-\mathrm{kDa}$ serine/threonine kinase, controls protein translation by the phosphorylation of downstream effectors: the
p70S6K that activates 4E-BP1, which in turn binds to eIF4E and inhibits eIF4E function. Hyperphosphorylation of 4E-BP1 disrupts this binding, releasing eIF4E to be phosphorylated at Ser 209. Phosphorylation of eIF4E increases its affinity for the cap of mRNA and may also favor its entry into initiation complexes $(27,28)$. In the ERK pathway, ERK1/2 can directly phosphorylate eIF4E at Ser $209(29,30)$. Thus, the inhibition of eIF4E phosphorylation by celastrol could play an important role in its downregulation of HIF-1 $\alpha$ protein synthesis. A number of studies indicate that deregulation of protein synthesis is a major contributor in cancer initiation and metastatic progression $(31,32)$. For example, eIF4E overexpression has been demonstrated in a variety of human tumors, and has been related to disease progression. Overexpression of eIF4E in experimental models markedly alters cellular morphology, enhances proliferation and induces cellular transformation, tumorigenesis and metastasis. Conversely, blocking eIF4E function by expression of antisense RNA, suppresses cellular transformation, tumor growth, tumor invasiveness and metastasis. It was reported that mTOR inhibitor rapamycin increased eIF4E phosphorylation through PI3K-dependent and MEK-mediated mechanisms, indicating mTOR-targeted cancer therapy may confer a resistance through a negative feedback mechanism $(33,34)$. In this sense, celastrol could be a valuable lead compound for further development of a new potent anticancer agent in combination with mTOR inhibitors.

In summary, this study shows, for the first time, that celastrol inhibits the mTOR/p70S6K/eIF4E and ERK signaling pathways, and HIF-1 activity in Hep3B cells. Thus, we have elucidated important mechanisms of the anticancer activity of celastrol, related to cell invasion and angiogenesis, which are essential for the adaptation of cancer cells to microenvironmental hypoxia and, hence, for tumor progression. These mechanisms may partly explain the broad spectrum of celastrol anticancer effects, and provide a rationale for the development of celastrol as an anticancer drug.

\section{Acknowledgements}

This study was supported by the National Natural Science Foundation of China, nos. 81160250 and 81360496 and was partially supported by the Jilin province Science and Technology Development Plan item, no. 20130101161JC. This study also received assistance from The Thousand Peoples Plan by the Foreign Expert Bureau, China.

\section{References}

1. Harris AL: Hypoxia - a key regulatory factor in tumour growth. Nat Rev Cancer 2: 38-47, 2002.

2. Semenza GL: HIF-1 and tumor progression: pathophysiology and therapeutics. Trends Mol Med 8: S62-S67, 2002.

3. Bruick RK and McKnight SL: A conserved family of prolyl4-hydroxylases that modify HIF. Science 294: 1337-1340, 2001.

4. Epstein AC, Gleadle JM, McNeill LA, et al: C. elegans EGL-9 and mammalian homologs define a family of dioxygenases that regulate HIF by prolyl hydroxylation. Cell 107: 43-54, 2001.

5. Ivan M, Kondo K, Yang $\mathrm{H}$, et al: HIFalpha targeted for VHL-mediated destruction by proline hydroxylation: implications for O2 sensing. Science 292: 464-468, 2001.

6. Jaakkola P, Mole DR, Tian YM, et al: Targeting of HIF-alpha to the von Hippel-Lindau ubiquitylation complex by $\mathrm{O}_{2}$-regulated prolyl hydroxylation. Science 292: 468-472, 2001. 
7. Jeong JW, Bae MK, Ahn MY, et al: Regulation and destabilization of HIF-1alpha by ARD1-mediated acetylation. Cell 111: 709-720, 2002

8. Lu X and Kang Y: Hypoxia and hypoxia-inducible factors: master regulators of metastasis. Clin Cancer Res 16: 5928-5935, 2010.

9. Mole DR, Blancher C, Copley RR, et al: Genome-wide association of hypoxia-inducible factor (HIF)-1alpha and HIF-2alpha DNA binding with expression profiling of hypoxia-inducible transcripts. J Biol Chem 284: 16767-16775, 2009.

10. Xia X, Lemieux ME, Li W, et al: Integrative analysis of HIF binding and transactivation reveals its role in maintaining histone methylation homeostasis. Proc Natl Acad Sci USA 106: 4260-4265, 2009.

11. Semenza GL: Targeting HIF-1 for cancer therapy. Nat Rev Cancer 3: 721-732, 2003.

12. Giaccia A, Siim BG and Johnson RS: HIF-1 as a target for drug development. Nat Rev Drug Discov 2: 803-811, 2003.

13. Belozerov VE and Van Meir EG: Hypoxia inducible factor-1: a novel target for cancer therapy. Anticancer Drugs 16: 901-909, 2005.

14. Salminen A, Lehtonen M, Paimela T and Kaarniranta K Celastrol: molecular targets of thunder god vine. Biochem Biophys Res Commun 394: 439-442, 2010.

15. Hervouet E, Cizkova A, Demont J, et al: HIF and reactive oxygen species regulate oxidative phosphorylation in cancer. Carcinogenesis 29: 1528-1537, 2008.

16. Jin X, Jin HR, Lee D, Lee JH, Kim SK and Lee JJ: A quassinoid 6alpha-tigloyloxychaparrinone inhibits hypoxia-inducible factor-1 pathway by inhibition of eukaryotic translation initiation factor 4E phosphorylation. Eur J Pharmacol 592: 41-47, 2008.

17. Jin HR, Jin SZ, Cai XF, et al: Cryptopleurine targets NF- $\kappa \mathrm{B}$ pathway, leading to inhibition of gene products associated with cell survival, proliferation, invasion, and angiogenesis. PLoS One 7: e40355, 2012.

18. Hagen T, Taylor CT, Lam F and Moncada S: Redistribution of intracellular oxygen in hypoxia by nitric oxide: effect on HIFlalpha. Science 302: 1975-1978, 2003.

19. Choi YK, Kim CK, Lee H, et al: Carbon monoxide promotes VEGF expression by increasing HIF-1alpha protein level via two distinct mechanisms, translational activation and stabilization of HIF-1alpha protein. J Biol Chem 285: 32116-32125, 2010.

20. Lee JH, Koo TH, Yoon H, et al: Inhibition of NF-kappa B activation through targeting I kappa B kinase by celastrol, a quinone methide triterpenoid. Biochem Pharmacol 72: 1311-1321, 2006.

21. Pang X, Yi Z, Zhang J, et al: Celastrol suppresses angiogenesismediated tumor growth through inhibition of AKT/mammalian target of rapamycin pathway. Cancer Res 70: 1951-1959, 2010.

22. Huang Y, Zhou Y, Fan Y and Zhou D: Celastrol inhibits the growth of human glioma xenografts in nude mice through suppressing VEGFR expression. Cancer Lett 264: 101-106, 2008.
23. Yang H, Chen D, Cui QC, Yuan X and Dou QP: Celastrol, a triterpene extracted from the Chinese "thunder of god vine," is a potent proteasome inhibitor and suppresses human prostate cancer growth in nude mice. Cancer Res 66: 4758-4765, 2006.

24. Laughner E, Taghavi P, Chiles K, Mahon PC and Semenza GL: HER2 (neu) signaling increases the rate of hypoxia-inducible factor 1alpha (HIF-lalpha) synthesis: novel mechanism for HIF-1-mediated vascular endothelial growth factor expression. Mol Cell Biol 21: 3995-4004, 2001.

25. Thomas GV, Tran C, Mellinghoff IK, et al: Hypoxia-inducible factor determines sensitivity to inhibitors of mTOR in kidney cancer. Nat Med 12: 122-127, 2006.

26. Hart S, Novotny-Diermayr V, Goh KC, et al: VS-5584, a novel and highly selective PI3K/mTOR kinase inhibitor for the treatment of cancer. Mol Cancer Ther 12: 151-161, 2013.

27. Lachance PE, Miron M, Raught B, Sonenberg N and Lasko P: Phosphorylation of eukaryotic translation initiation factor $4 \mathrm{E}$ is critical for growth. Mol Cell Biol 22: 1656-1663, 2002.

28. Fukuda R, Hirota K, Fan F, Jung YD, Ellis LM and Semenza GL: Insulin-like growth factor 1 induces hypoxia-inducible factor 1-mediated vascular endothelial growth factor expression, which is dependent on MAP kinase and phosphatidylinositol 3-kinase signaling in colon cancer cells. J Biol Chem 277: 38205-38211, 2002.

29. Ueda T, Watanabe-Fukunaga R, Fukuyama H, Nagata S and Fukunaga R: Mnk2 and Mnk1 are essential for constitutive and inducible phosphorylation of eukaryotic initiation factor $4 \mathrm{E}$ but not for cell growth or development. Mol Cell Biol 24: 6539-6549, 2004.

30. Liu LP, Ho RL, Chen GG and Lai PB: Sorafenib inhibits hypoxia-inducible factor-1alpha synthesis: implications for antiangiogenic activity in hepatocellular carcinoma. Clin Cancer Res 18: 5662-5671, 2012.

31. Bjornsti MA and Houghton PJ: Lost in translation: dysregulation of cap-dependent translation and cancer. Cancer Cell 5: 519-523, 2004.

32. De Benedetti A and Graff JR: eIF-4E expression and its role in malignancies and metastases. Oncogene 23: 3189-3199, 2004.

33. Wang X, Yue $\mathrm{P}$, Chan CB, et al: Inhibition of mammalian target of rapamycin induces phosphatidylinositol 3-kinase-dependent and Mnk-mediated eukaryotic translation initiation factor 4E phosphorylation. Mol Cell Biol 27: 7405-7413, 2007.

34. Wendel HG, De Stanchina E, Fridman JS, et al: Survival signalling by Akt and eIF4E in oncogenesis and cancer therapy. Nature 428: 332-337, 2004. 